\title{
Expression of pericardial fluid T-cells and related inflammatory cytokines in patients with chronic heart failure
}

\author{
REINARD ISKANDAR * , SHENGCHEN LIU* , FEI XIANG, WEN CHEN, \\ LIANGPENG LI, WEI QIN, FUHUA HUANG and XIN CHEN \\ Department of Thoracic and Cardiovascular Surgery, Nanjing First Hospital, \\ Nanjing Medical University, Nanjing, Jiangsu 210006, P.R. China
}

Received December 6, 2015; Accepted December 19, 2016

DOI: $10.3892 /$ etm.2017.4202

\begin{abstract}
Pericardial fluid, as a biochemical indicator of heart status, directly indicates pathological alteration to the heart. The accumulation of pericardial fluid can be attributed to an underlying systemic or local inflammatory process. However, the pericardial fluid expression of cellular surface markers, as well as several cytokines in chronic heart failure (CHF), remain unclear. In order to evaluate these issues further the pericardial fluid expression of several cytokines and the surface expression of activity markers between CHF patients and non-heart failure (NHF) patients were analyzed. The pericardial fluid expression of cytokines was measured by immunofluorescence and biomarker of plasma N-terminal propeptide of B-type natriuretic peptide (NT-proBNP), while pericardial fluid levels of soluble glycoprotein 130 (sgp130) were analyzed by ELISA in $50 \mathrm{CHF}$ and $24 \mathrm{NHF}$ patients. In addition, the surface expression of activation markers for T-cells was measured by immunohistochemistry. Patients with CHF demonstrated increased levels of plasma NT-proBNP and pericardial fluid sgp130. Surface expression of cellular activation markers CD25 and Foxp3 in the pericardial fluid was increased in patients with CHF. Moreover, the pro- and anti-inflammatory cytokines interferon (IFN)- $\gamma$, interleukin (IL)-6 and IL-10 in patients with CHF also demonstrated an increased expression within its pericardial fluid. In addition, there was infiltration of inflammatory cells and enhanced expression of inflammatory cytokines in the pericardial fluid of patients with $\mathrm{CHF}$, which may reflect $\mathrm{T}$ cell activation, suggesting that systemic inflammation is important in the
\end{abstract}

Correspondence to: Professor Xin Chen, Department of Thoracic and Cardiovascular Surgery, Nanjing First Hospital, Nanjing Medical University, 68 Changle Road, Nanjing, Jiangsu 210006, P.R. China

E-mail: stevecx@njmu.edu.cn

*Contributed equally

Key words: pericardial fluid, $\mathrm{T}$ cells, cytokines, inflammation, chronic heart failure progression of CHF. This evidence could indicate a possible novel target for future therapeutics and prevention of $\mathrm{CHF}$.

\section{Introduction}

Chronic heart failure (CHF) has currently become a global epidemic with high prevalence, incidence and mortality, and is one of the deadliest diseases (1). It is diagnosed as a state of chronic inflammation with elevated T-cell activation and inflammatory cytokine production in the circulatory system (2). A recent study revealed strong evidence that $\mathrm{T}$ lymphocytes are important in the pathogenesis of cardiac disease (3). In addition, inflammatory cytokines have been investigated as targets of heart failure therapy (4).

The development and progression of CHF itself are closely associated with the imbalance between pro- and anti-inflammatory mediators, and patients with $\mathrm{CHF}$ are characterized by an increased expression of inflammatory mediators in circulating leukocytes. For example, using blood samples, T-lymphocytes of patients with $\mathrm{CHF}$ demonstrated increased expression of interferon (IFN) and interleukin (IL)-10 in addition to increased surface activation markers, including CD69 and CD25 (5). Hence, IFN- $\gamma$ and IL-10 have been considered to be candidate cytokines involved in the pathomechanism leading to CHF (6). Another surface marker, the forkhead/winged helix transcription factor (Foxp3), which is expressed by regulatory $\mathrm{T}$ cells (Treg), has an anti-inflammatory role and maintains tolerance to self-components by contact-dependent suppression or by releasing anti-inflammatory cytokines, including IL-10 (7). Within the blood, IL-10 itself has potent deactivating properties in macrophages and T-cells and thus acts as a downregulator of cell-mediated immune responses, which have already been described as being important in CHF (8).

Circulating inflammatory markers, including IL-6, may be useful in establishing a diagnosis and in gauging a prognosis in patients with heart failure (HF) (4). IL-6 is a multifunctional cytokine that mediates both immune and inflammatory responses. It has been shown that increased plasma IL-6 levels may contribute to disease progression, and may be associated with enhanced mortality in patients with CHF (9). Within the myocardium, IL-6-type cytokines convey their signals predominantly through the signal transducer and 
activator of the transcription 3 pathway via a glycoprotein 130 (gp130) receptor subunit. Moreover, the circulating levels of the common receptor subunit, soluble gp130 (sgp130), may potentially indicate the activity of the whole IL-6 family, including myocardial activation. It is the common receptor of IL-6, which is elevated in patients with CHF (10).

Pericardial fluid may be used as a biomarker for inferring pathological alteration to the heart (11), and its accumulation can be attributed to an underlying systemic or local inflammatory process (12). Therefore, it is important to identify the association between the progression of $\mathrm{CHF}$ and the inflammatory response in the pericardial fluid in order to find a future treatment strategy and prognosis of HF. In order to further evaluate the role of pericardial fluid in CHF patients, the pericardial fluid expression of several cytokines and also surface expression of activity markers in T-cells from $\mathrm{CHF}$ patients and controls was examined.

\section{Materials and methods}

Patients. The present study population consisted of 74 patients with various degrees of $\mathrm{CHF}$ who were undergoing cardiac surgery in Nanjing First Hospital (Nanjing, China). Diagnosis of the etiology of HF was based on the clinical history, symptoms, physical examination, echocardiography, chest $\mathrm{X}$-rays, electrocardiography and cardiac catheterization, conforming to available guidelines regarding $\mathrm{CHF}(13,14)$. Echocardiographic evaluation was performed according to the guidelines of the American Society of Echocardiography (15) before cardiac surgery using a commercially available probe and system to assess cardiac function and measure the left ventricular ejection fraction. Coronary angiography was performed in all patients before surgery to exclude patients with coronary artery disease. The following laboratory tests were also performed within the first $24 \mathrm{~h}$ after admission: Complete blood count, tests of liver function, measurements of blood urea nitrogen and serum creatinine, body mass index (BMI) and serum electrolytes.

Patients meeting the criteria for the present study were prospectively assigned to the $\mathrm{CHF}$ or NHF group. Moreover, the functional severity of HF was assessed prior to the surgery using the New York Heart Association (NYHA) classification $(16,17)$, and the patients were classified by an independent investigator as: i) Symptomatic, which had a history or presence of clinical symptoms and sign of CHF including, orthopnea, paroxysmal nocturnal dyspnea, pulmonary congestion on chest radiograph or a significant exertional dyspnea or fatigue (NYHA II, n=26; NYHA III, n=21; NYHA IV, n=3) for the CHF group or ii) asymptomatic (NYHA I, n=24) for the NHF group. Patients were excluded if they had a history of recent myocardial infarction or unstable angina in the last 12 months. Other exclusion criteria included acute coronary syndrome, end-stage renal failure, shock, cancer and severe infection. Moreover, the study conformed to the principles outlined in the Declaration of Helsinki, the Ethics Committee of Nanjing Medical University Affiliated Nanjing First Hospital approved the trial, and each patient provided written informed consent.

Data collection. Sociodemographics (i.e., gender, age, education, marital status, living status and employment status) and information pertaining to medical history (i.e. CHF etiology and co-morbidities, including dyslipidemia and chronic kidney disease) were collected. Information about medication use, history of hypertension, diabetes mellitus, coronary heart disease, chronic obstructive pulmonary disease and stroke were also obtained. The baseline characteristics of the patient demographic variables in the present study, HF classification and echocardiographic parameters of patients are shown in Table I.

Measurement of biomarkers. Pericardial fluid samples were obtained during surgery under general anesthesia. Undiluted samples of the pericardial fluid were obtained immediately after the creation of a small incision in the pericardium and before heparinization. Samples contaminated with blood were discharged. Pericardial fluid was collected for the measurement of total protein. Arterial blood samples were withdrawn from the cannulated brachial artery at the same time for the measurement of plasma N-terminal propeptide of B-type natriuretic peptide (NT-proBNP). In addition, all the samples were collected into sterile tubes and immediately placed on ice. These were then immediately centrifuged at 3,000 $\mathrm{x} g$ for $15 \mathrm{~min}$ at $4^{\circ} \mathrm{C}$ and rapidly frozen and stored at $-80^{\circ} \mathrm{C}$ until further analysis.

Histological analysis. Cells obtained from the pericardial fluid samples were fixed in $4 \%$ paraformaldehyde (PFA) smear section, which was stained with hematoxylin and eosin (H\&E) to assess the cell proportions within each group for histopathology. Stained sections were performed according to a previously described protocol with minor revisions (18), and were then observed under a BX41TF microscope (Olympus Corporation, Tokyo, Japan). True color digital images were then captured.

Immunohistochemical staining. The expression levels of Foxp3 and CD25 receptor protein were examined immunohistochemically in pericardial fluid biopsy samples obtained from the patients with CHF and NHF. Staining of CD25 (1:100; ab61195, Abcam, Cambridge, MA, USA) and Foxp3 (1:100; sc-28705, Santa Cruz Biotechnology Inc., Santa Cruz, CA, USA) immunohistochemistry was performed on formalin-fixed sections, followed by incubation with PV-6001 (Beijing Zhongshan Golden Bridge Biotechnology Co., Ltd., Beijing, China) according to the manufacturer's instructions. Each stained histological section was examined under an Olympus BX53F microscope (Olympus Corporation) connected to a computerized image-analysis system (Image-Pro v6.0; Media Cybernetics Inc., Silver Spring, MD, USA).

Immunofluorescence staining. Immunofluorescence staining for IL-6 (1:100; ab6672; Abcam, Cambridge, MA, USA), IL-10 (1:100; 250713; Abbiotec, San Diego, CA, USA) and IFN- $\gamma$ (1:100; ab175878; Abcam) was performed in a smear section. Prior to immunostaining, cells were washed with phosphate-buffered saline, fixed with 4\% PFA for $10 \mathrm{~min}$ and permeabilized for $10 \mathrm{~min}$ using $0.05 \%$ Triton-100. Cells were then incubated for $20 \mathrm{~min}\left(37^{\circ} \mathrm{C}\right)$ with $5 \%$ bovine serum albumin blocking solution in order to block non-specific binding. Cells were then incubated at $4^{\circ} \mathrm{C}$ overnight with 
primary antibodies and $1 \mathrm{~h}$ with the appropriate secondary antibody conjugated to Alexa-488 (1787900) or Alexa 594 (1827987; both Invitrogen; Thermo Fisher Scientific, Inc., Carlsbad, CA, USA). DAPI was used to counterstain the nuclei. Samples were then covered with mounting media (Invitrogen; Thermo Fisher Scientific, Inc.), overlaid with coverslips and examined under a fluorescence microscope (Axio Scope A1; Carl Zeiss AG, Oberkochen, Germany) following the method described in previous studies $(19,20)$.

ELISA. The concentration of NT-proBNP and Sgp130 levels were evaluated using the highly sensitive ELISA. NT-proBNP was measured by the ELISA kit for human NT-proBNP (Cloud-Clone Corp, Houston, TX, USA). Sgp130 was measured using a human sgp130 Quantikine ELISA kit (R\&D Systems, Inc., Minneapolis, MN, USA) all following the manufacturer's instructions. The assays were quantified in an ultra-microplate reader at a wavelength of $450 \mathrm{~nm}$ (ELX808; BioTek Instruments, Inc., Winooski, VT, USA). The results are expressed as $\mathrm{pg} / \mathrm{ml}$.

Statistical analysis. Comparisons of CHF patients vs. control subjects were performed using Student's $t$-test where distributions of data were normal and homogeneity of variance. For skewed variables the Mann-Whitney U test was used for the analysis. For the ranked data, Pearson's $\chi^{2}$ or Fisher's exact tests were used for the comparison between two groups. Continuous values are expressed as the mean \pm standard deviation. Data were analyzed using SPSS version 13.0 (SPSS, Inc., Chicago, IL, USA). $\mathrm{P}<0.05$ was used to indicate a statistically significant difference.

\section{Results}

Clinical characteristics in CHF patients. In total, 74 patients were enrolled in the present study. The baseline clinical characteristics of the study subjects are detailed in Table I, which summarizes the demographic data, medical history, NYHA function classification and BMI in the CHF and NHF groups. There were no significant differences in age, gender and BMI between the two groups. Moreover, according to the NYHA classification, the severity of HF was class I in 24, class II in 26 , class III in 21 and class IV in three patients.

Concentration of plasma NT-proBNP in patients with CHF. Plasma NT-pro BNP was observed in CHF and NHF patients. The level of plasma NT-proBNP significantly increased in the CHF group compared to the NHF group (749.60 \pm 468.06 vs. $303.45 \pm 275.00 \mathrm{pg} / \mathrm{ml} ; \mathrm{P}<0.05$ ) (Fig. 1).

Pericardial fluid expression in cellular proportion of patients with $C H F$. The pericardial fluid of CHF patients exhibited marked infiltration of inflammatory cells, whereas the pericardial fluid of the NHF patients had less of such infiltrates. Moreover, CHF patients showed an increased number of circulating lymphocytes within its pericardial fluid compared to NHF patients as evidenced by H\&E staining (Fig. 2).

Pericardial fluid expression on surface markers CD25 and Foxp3. In order to further elucidate the role of lymphocyte
Table I. Baseline characteristics in non-heart failure patients and in patients with chronic heart failure.

\begin{tabular}{lcc}
\hline Characteristics & NHF $(\mathrm{n}=24)$ & CHF $(\mathrm{n}=50)$ \\
\hline Age (years) & $57.79 \pm 9.81$ & $57.30 \pm 10.92$ \\
Gender (male/female) & $11 / 13$ & $21 / 29$ \\
Body mass index $\left(\mathrm{kg} / \mathrm{m}^{2}\right)$ & $22.98 \pm 3.64$ & $24.01 \pm 3.57$ \\
New York Heart Association & & \\
I & 24 & - \\
II & - & 26 \\
III & - & 21 \\
IV & - & 3 \\
Diabetes mellitus, n $(\%)$ & $1(4)$ & $2(4)$ \\
COPD, $\mathrm{n}(\%)$ & - & $1(2)$ \\
Stroke, $\mathrm{n}(\%)$ & - & $2(4)$ \\
Echocardiographic data & & \\
LVEF $(\%)$ & $64.21 \pm 1.98$ & $69.04 \pm 17.21$ \\
Creatinine (mmol/l) & $62.19 \pm 18.76$ & $69.04 \pm 17.21$ \\
\hline
\end{tabular}

Data are presented as the mean \pm standard deviation, or number or percentage of CHF patients or NHF patients. NHF, non-heart failure; $\mathrm{CHF}$, chronic heart failure; COPD, chronic obstructive pulmonary disease; LVEF, left ventricular ejection fraction.

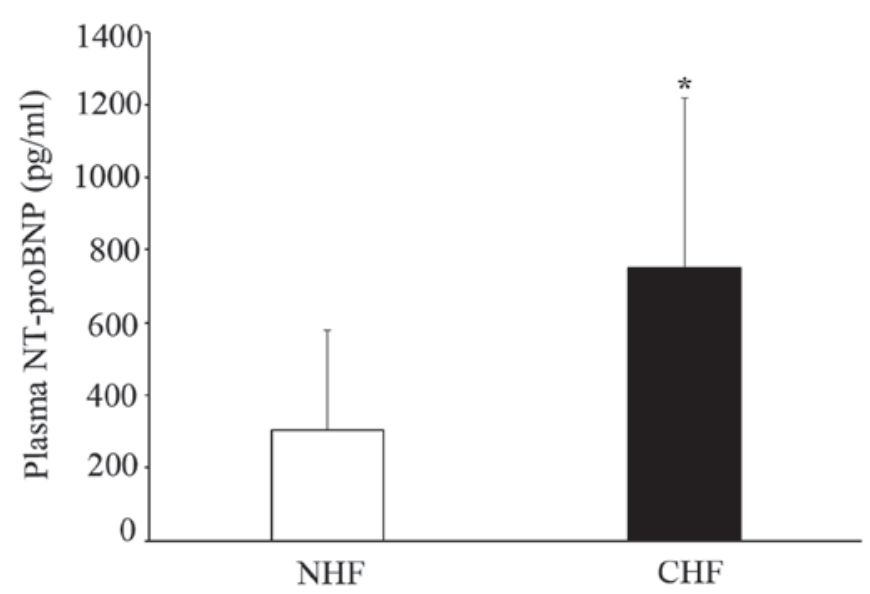

Figure 1. Expression of plasma NT-proBNP levels from NHF $(n=24)$ and CHF ( $\mathrm{n}=50$ ) patients. ${ }^{*} \mathrm{P}<0.05$ vs. NHF. NT-proBNP, N-terminal propeptide of B-type natriuretic peptide; NHF, non-heart failure; CHF, chronic heart failure.

cells in the systemic inflammatory response during CHF, the surface expression of the $\mathrm{T}$ cell activation markers, CD25 and Foxp3, on pericardial fluid were detected using immunohistochemistry. The T-cell activation marker CD 25 was observed to be significantly increased in CHF patients compared to the controls $(\mathrm{P}<0.05$; Fig. 3$)$. Foxp3 staining by immunohistochemistry also revealed that there was an enhanced expression of cells present in the pericardial fluid of CHF patients compared with NHF patients. The quantitative analysis of the percentage of positive cells ( $\mathrm{n}=100$ cells each) was also measured by immunostaining. The proportion of $\mathrm{CD} 25$-positive cells was significantly higher in the CHF group 

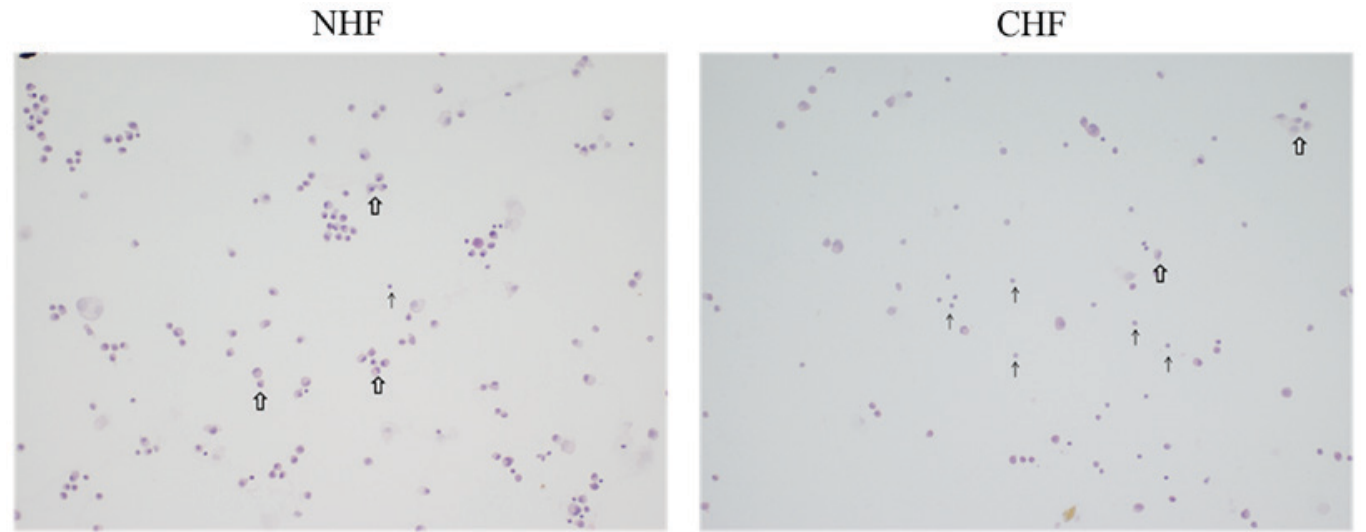

Figure 2. Pericardial fluid stained of NHF and CHF patients shown by hematoxylin and eosin staining. CHF patients revealed increased number of lymphocyte infiltration (magnification, $\mathrm{x} 40$ ). Black arrows indicate lymphocyte cells and white arrows indicate epithelial cells. NHF, non-heart failure; CHF, chronic heart failure.
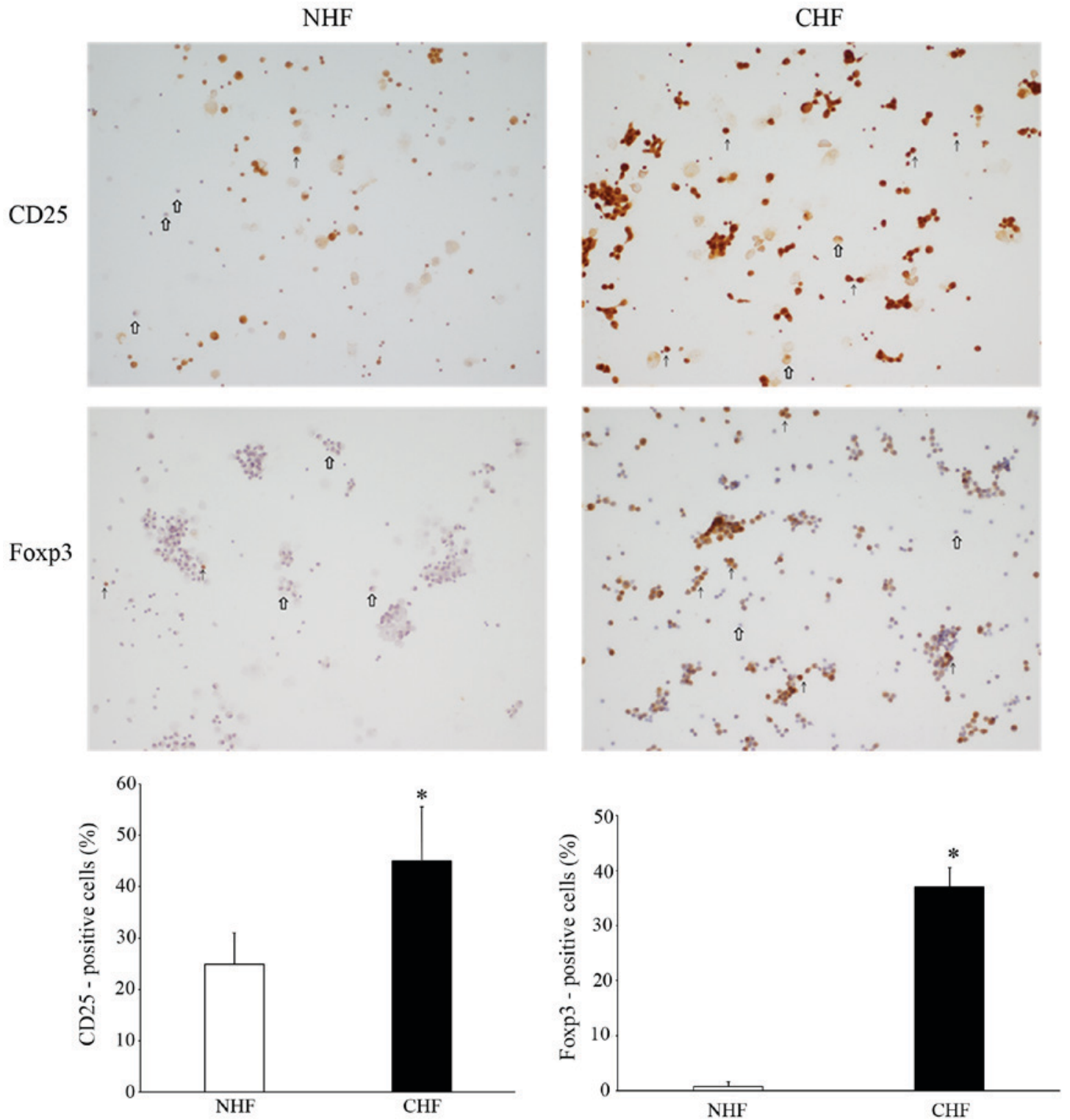

Figure 3. Different degrees of immunostaining for surface expression in pericardial fluid samples obtained from patients with CHF and NHF. Representative picture demonstrates the distribution of CD25 T lymphocytes infiltration and Foxp3 within pericardial fluid of CHF patients identified by immunohistology (black arrows indicate lymphocyte and white arrows indicate epithelial cells). The figure also indicates the percentage of CD25 and Foxp3 positive cells respectively measured by immunostaining ( $\mathrm{n}=100$ cells each). " $\mathrm{P}<0.05$ vs. NHF. NHF, non-heart failure; CHF, chronic heart failure; Foxp3, forkhead/winged helix transcription factor. 

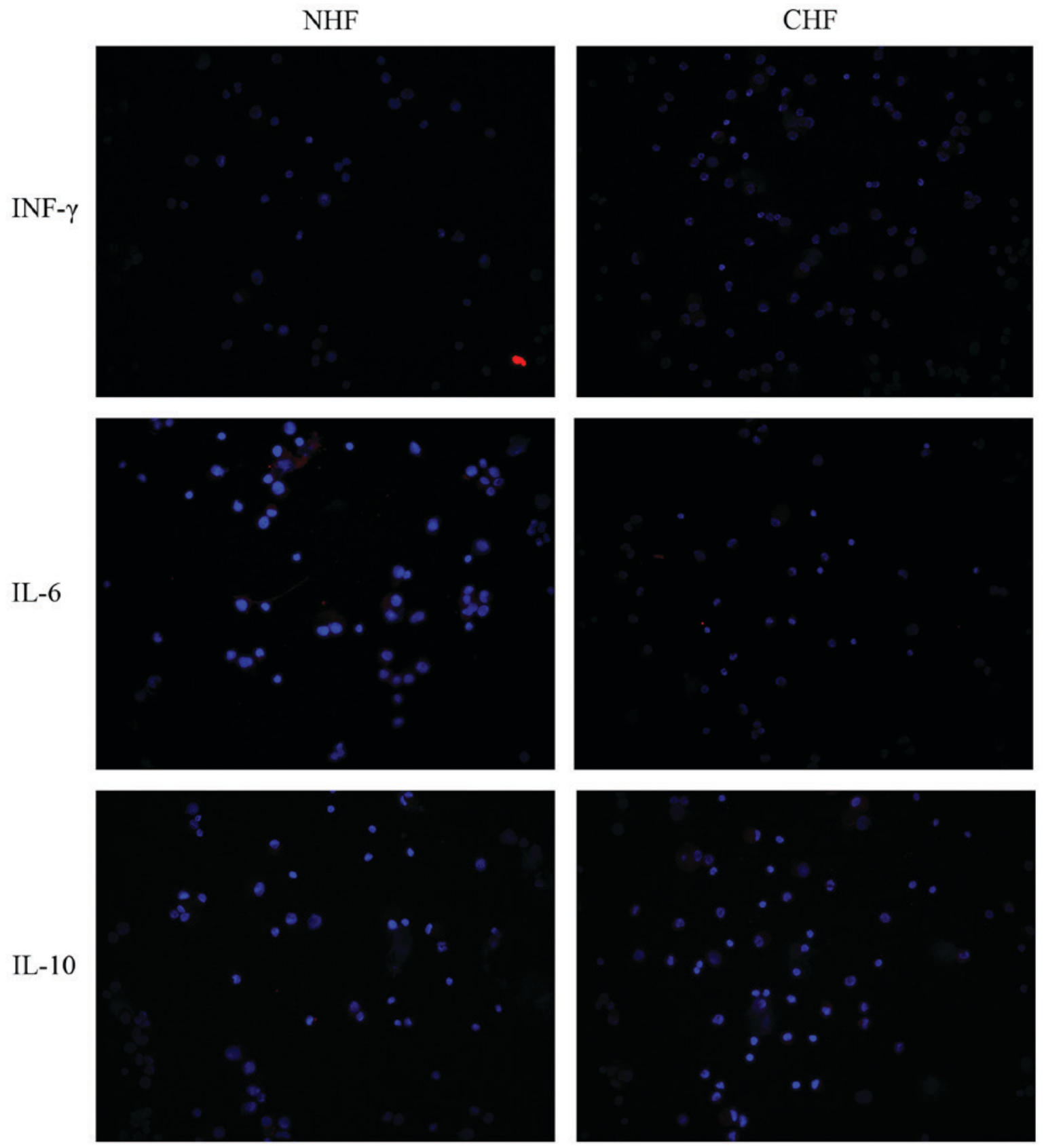

Figure 4. Pericardial fluid expression of pro- and anti-inflammatory cytokines in patients with CHF and NHF visualized by fluorescence microscope (magnification, $\mathrm{x} 20$ ). The cells express interferon- $\gamma$, interleukin- 6 and IL-10 within pericardial fluid (blue indicates staining; red, indicates signal). Enhanced expression of IFN- $\gamma$ was shown in the pericardial fluid of CHF patients. IL-6 showed an increased expression in the pericardial fluid of CHF patients. Pericardial fluid expression of IL-10 was also increased in CHF patients. CHF, chronic heart failure; NHF, non-heart failure; IFN, interferon; IL, interleukin.

compared to the NHF group $(45.00 \pm 10.58$ vs. $24.92 \pm 6.13 \%$; $\mathrm{P}<0.05$ ); while Foxp3-positive cells were also significantly higher in the CHF group compared to the NHF group $(36.97 \pm 3.50 \%$ vs. $0.76 \pm 0.90 \%$; $\mathrm{P}<0.05)$ (Fig. 3 ), suggesting $\mathrm{T}$ cell activation within the pericardial fluid of the CHF patients.

Pericardial fluid expression of the pro- and anti-inflammatory cytokines IFN- $\gamma$, IL- 6 and IL-10. Given the inflammatory reactions in the hearts of CHF patients, it was of interest to examine whether pro- and anti-inflammatory cytokines were detectable in the pericardial fluid of such patients. Therefore, the present study investigated the pericardial fluid expression of IFN- $\gamma$,
IL-6 and IL-10. The pro-inflammatory cytokine expression of the pericardial fluid IFN- $\gamma$ was markedly increased in the CHF compared to the NHF patients. Moreover, pericardial fluid expression of IL-6 was also markedly increased in the CHF compared with the NHF patients. On the other hand, pericardial fluid expression of the anti-inflammatory cytokine IL-10 was also showing a markedly increase in cells of CHF compared to NHF patients (Fig. 4).

Circulating levels of sgpl30 in the pericardial fluid of patients with CHF. Finally, the pericardial fluid expression of the IL-6 receptor sgp130 levels in patients with CHF was examined in the present study. Patients with CHF had significantly increased 


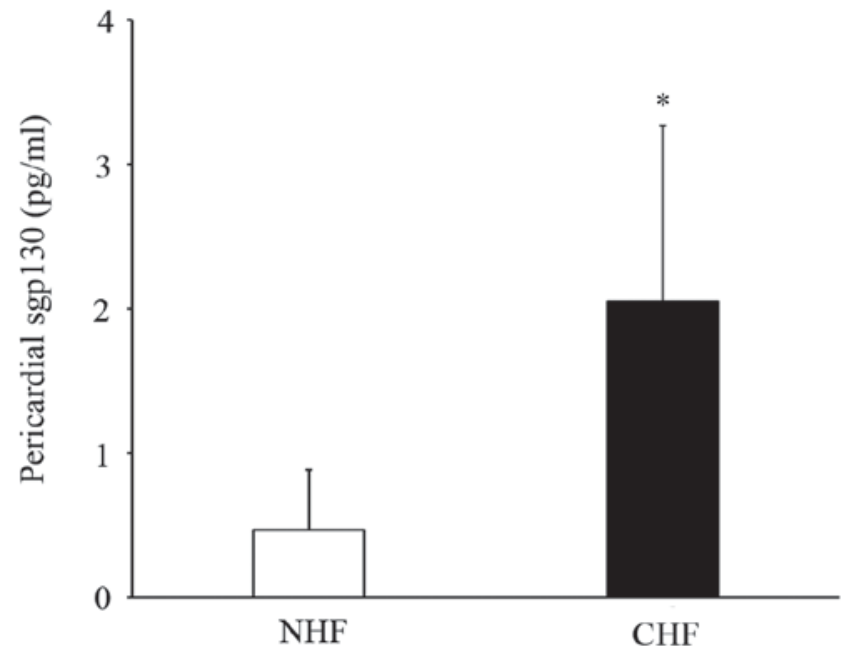

Figure 5. Expression of pericardial sgp130 levels from NHF and CHF patients. ${ }^{*} \mathrm{P}<0.05$ vs. NHF. NHF, non-heart failure; $\mathrm{CHF}$, chronic heart failure.

levels of pericardial sgp130 fluid compared with NHF patients $(2.05 \pm 1.21$ vs. $0.46 \pm 0.40 \mathrm{pg} / \mathrm{ml} ; \mathrm{P}<0.05)$ (Fig. 5).

\section{Discussion}

$\mathrm{CHF}$ is an increasingly prevalent disease, which claims thousands of lives every year (21). Previous studies have identified some prognostic markers indicating adverse outcomes in patients with CHF (22-24). However, researchers have still not reached a consensus on the incidence, characteristics and importance of pericardial fluid in patients with CHF. To the best of our knowledge, the present study investigated for the first time the expression of pericardial fluid $\mathrm{T}$ cells and the related inflammatory cytokines in $\mathrm{CHF}$ patients.

NT-proBNP has recently become a subject of interest amongst medical researchers due to its possible role in monitoring HF and distinguishing acute coronary syndromes (25). It has recently been reported that plasma, fresh and frozen urine levels of NT-proBNP were significantly higher in CHF patients (26). A previous study suggests that the severity of CHF could be determined on the basis of plasma NT-proBNP levels, whereas the NT-proBNP assay may be more sensitive than BNP under certain circumstances (27). Therefore, in the present study the NT-proBNP levels were evaluated. These were used to confirm the diagnosis of CHF in the population of the present study, regardless of history, etiology and symptoms of CHF itself. In agreement with previous results (26-28), the plasma levels of NT-proBNP in the present study were significantly increased in patients with $\mathrm{CHF}$, suggesting that NT-proBNP is a useful marker for detecting CHF.

HF occurs due to the inability of the heart to respond to the circulatory demand; main causes include ischemic and valvular injuries, while toxic, metabolic or genetic (congenital) origins are less common. Alterations in the heart may lead to major changes in the myocardial structure and function. The pathogenesis of HF itself is determined by numerous factors, including inflammation, neuroendocrine activation, oxidative stress, severe angiogenesis, apoptosis pathway changes and vascular remodeling (29). Inflammation may stimulate cardiac remodeling and fibrosis, and thus participates in the progression and pathogenesis of HF. Gruson et al (30) reported that circulating levels of cytokines are enhanced in the failing myocardium, and increased production of pro-inflammatory cytokines may challenge the surrounding tissue through propagation of the inflammatory response and direct effects on the cardiac myocyte structure and its function. Pro-inflammatory cytokines such as the tumor necrosis factor- $\alpha$ and IL family (IL-6, IL-1 and IL-18) appear to cause cardiomyocyte apoptosis and necrosis as well as cell hypertrophy, leading to CHF (30). In the present study, which investigated pericardial fluid samples, an aim was to detect biomarkers of inflammation associated with $\mathrm{CHF}$ that may provide additional options, rather than NT-proBNP alone, for diagnosing CHF. There was a notable limitation within our study. Although the concentration of plasma NT-proBNP increased in the CHF group, the standard deviation of these data are quite large, which may have been caused by a deficiency of the samples. Furthermore, although we have attempted to decrease the influence of other clinical factors, there are still some factors that we did not recognize that may affect the results.

Activation of the immune system and inflammation in $\mathrm{HF}$ patients are considered to be important in the progression of HF (22,31-33). In particular, variations in different types of leukocytes, including lymphocyte, monocyte, eosinophil and mast cells, have been identified in a high-risk subset of HF patients (34). It has been hypothesized that the typical hallmarks for the involvement of immune mechanisms in $\mathrm{CHF}$ pathogenesis are the infiltration of cardiac tissue by leukocytes (6). We hypothesized that $\mathrm{T}$ cell infiltration will also appear within the pericardial fluid of CHF patients, the present results demonstrated that $\mathrm{CHF}$ patients had an increasing number of circulating $\mathrm{T}$ lymphocytes within its pericardial fluid compared to NHF patients, which indicates increased $\mathrm{T}$ cell infiltration within the pericardial fluid of CHF patients.

$\mathrm{T}$ cells in CHF patients are evidently activated, as evidenced by enhanced gene expression of chemokines and inflammatory cytokines, in addition to the surface expression of activated markers (35). CD25 T lymphocytes represent the most well characterized subset of regulatory $\mathrm{T}$ cells on their surface, and recently, nuclear transcription factor Foxp3 has been demonstrated to a specific marker for and to regulate the development and function of $\mathrm{CD} 4^{+} \mathrm{CD} 25^{+}$Tregs (36). A previous publication stated that circulating Treg cells were reduced and their function was altered in CHF patients, regardless of etiology, indicating that the defects in Treg cells are responsible for the aberrant chronic immune activation in CHF patients (2). In the present study, the result demonstrated that T-cells in the pericardial fluid of CHF patients expressing CD25 and Foxp3 activation markers were significantly increased compared to the NHF patients. This observation was consistent with other reports $(37,38)$ that showed that T-cells from CHF patients had an enhanced surface expression of the activation marker CD25. This study demonstrated that circulating T-cells are markedly activated in CHF as assessed by both enhanced mRNA levels of several inflammatory cytokines as well as by an increased surface expression of activation markers (37). Moreover, the results of the present study suggested that CD25 is not only expressed within T lymphocytes but also within other cells, including monocytes. However, the present study focused on $\mathrm{T}$ lymphocytes that expressed the surface marker CD25. 
Other studies also reported that among the leukocyte subsets, monocytes have been shown to contribute to the systemic inflammation in HF patients $(38,39)$, and through this study it was suggested that $\mathrm{T}$ cells may be an important cellular source for inflammatory cytokines in CHF. However, the expression and functional role of CD25 within other cell types requires further study.

Besides monocyte function being modulated in $\mathrm{CHF}$ enhanced expression of pro-inflammatory cytokines and activation markers of T-cells has also been reported. However, in the present study, which based on the pericardial fluid sample, the results showed that pro-inflammatory cytokine expression of the pericardial fluid IFN- $\gamma$ was significantly increased in CHF compared to the NHF patients. There is clinical evidence that $\mathrm{T}$ cellular IFN- $\gamma$ gene expression is increased in patients with CHF (6). Cappuzzello et al (40) have described for the first time, that there was an increase of IL-9, and a decrease of IL-5, IL-7 and IFN- $\gamma$ plasma levels in patients with CHF. Moreover, Fukunaga et al (41) demonstrated that in $\mathrm{CHF}$ patients more $\mathrm{CD}^{+}{ }^{+}$-lymphocytes produce IFN, and that the number of $\mathrm{CD}^{+}$IFN producing T-lymphocytes increases with disease activity. IFN- $\gamma$ may protect against the development of dilated cardiomyopathy, HF and the incidence of mortality by reducing mast cell degranulation in the pericardium and preventing the adhesive, fibrous form of pericarditis (42).

During the last few years, an interest in the involvement of inflammatory factors in HF has emerged. The role of inflammation in the pathogenesis of HF has been strengthened with the implication of several cytokines, including IL-6, in the disease process in some experimental studies and the demonstration of elevated inflammatory markers in patients with milder degrees of HF, including those with asymptomatic left ventricular dysfunction as well as in patients at risk to develop HF (32). In the present study, the results demonstrated that pericardial fluid expression of IL- 6 was also significantly increased in CHF patients. Recently, a study by Hilfiker-Kleiner et al (43) has raised the warning that continuous gp130-mediated signal transducer and activator of transcription 3 (STAT3) activation promotes inflammation, left ventricular rupture and adverse remodeling in myocardial infarction. Moreover, these authors hypothesized that the elevation of IL-6 levels caused continuous activation of the gp130/STAT3 pathway, leading to HF. In addition, the serum IL-6 level is increased in patients with HF. Furthermore, Kinugawa et al (44) reported that cardiac expression of IL-6 mRNA is also increased in the myocardium in patients with advanced HF. IL- 6 may contribute to the progression of myocardial damage and dysfunction in the CHF syndrome (23). For several reasons, it is possible that the enhanced IL-6 activity in the failing myocardium may contribute to the increased gp130 and IL-6 levels in patients with CHF.

In patients with $\mathrm{HF}$, a decrease in IL-10 plasma concentration has been reported and it is positively correlated with a decrease in the left ventricular ejection fraction (45). In the present study, the results revealed that the anti-inflammatory cytokine IL-10 was increased in T-cells from CHF patients within its pericardial fluid compared to NHF patients. This result is supported by a previous study performed by Amir et al (46), which reported that the IL-10 circulating levels are higher in HF patients compared with healthycontrols. Moreover, Yndestad et al (39) reported a rise in inflammatory mediators that appears not to be accompanied by a corresponding increase in anti-inflammatory cytokines, including IL-10 in HF patients, and resulting in an inflammatory effect. Moreover in the present study, pericardial fluid expression of both pro- and anti-inflammatory mediators demonstrated cellular infiltrations of abundant T-cells within the pericardial fluid of CHF patients. This result suggests that the possible interaction in the complex of inflammatory and anti-inflammatory network may require further study.

It has previously been demonstrated that elevated sgp130 levels are associated with cardiovascular damage, and mortality resulting from worsening of HF (47). In the present study, the results showed that patients with CHF had significantly increased levels of pericardial fluid sgp130 compared with NHF patients, indicating that sgp130 may be involved in the progression of CHF. It has also been reported $(48,49)$ that elevated serum levels of IL-6 cytokines and gp130 proteins are strong prognostic markers for morbidity. Askevold et al (22) demonstrated that serum sgp130 was associated with fatal outcomes in patients with chronic systolic HF of ischemic causes, but did not appear to predict vascular events, and sgp130 did not enhance risk prediction when troponin $\mathrm{T}$ was accounted for and mortality in patients with HF or after myocardial infarction. gp130 is the common signal-transducing receptor subunit of the IL-6 family, which may be involved in the progression of HF. Sgp130 itself reflecting inflammatory processes, which has been reported is elevated in CHF patients (10). Moreover, the gp130 receptor system is important in the transition of left ventricular hypertrophy to overt HF (49).

It has been reported that increased levels of pro-inflammatory cytokines, in response to myocardial damage, are important prognostic factors correlating with increased mortality rates in HF patients (29). In addition, another study has indicated that the assessment of markers associated with the different pathways of HF pathogenesis may facilitate diagnosis and the prediction of mortality risk in patients with HF based on five years of observation (50). From this study it may be concluded that cytokines are increased in the pericardial fluid of the CHF, which may induce alteration in the structural matrix of the myocardium, and cause myocyte apoptosis leading to the inflammatory response, which may reflect $\mathrm{T}$ cell activation. Therefore, more data on the risk of stratification and therapy using presented biomarkers are required, and may warrant further studies and with larger populations.

In conclusion, to the best of our knowledge the present study reported for the first time that there is infiltration of inflammatory cells and enhanced expression of inflammatory cytokines within the pericardial fluid of patients with CHF, which reflects $\mathrm{T}$ cell activation. Moreover, it confirmed that the progression of CHF is associated with systemic inflammation. Furthermore, this evidence could represent a possible novel target that may lead to an improved understanding and future therapeutics and prevention to improve the clinical outcome in patients with $\mathrm{CHF}$.

\section{Acknowledgements}

The authors thank all the participants and people who worked in the present study. Dr Xin Chen is a fellow at the 
Collaborative Innovation Center for Cardiovascular Disease Translational Medicine. The present study was supported by grants from the National Science Foundation of China (grant no. 81370259) and the Clinical Scientific Grant of Jiangsu Province (grant no. BE2015621).

\section{References}

1. Zhu ZF, Li JJ, Liu J, Tang TT, Ding YJ, Liao YH, Cheng X and Wang X: Circulating Th17 cells are not elevated in patients with chronic heart failure. Scand Cardiovasc J 46: 295-300, 2012.

2. Tang TT, Zhu ZF, Wang J, Zhang WC, Tu X, Xiao H, Du XL, Xia JH, Dong NG, Su W, et al: Impaired thymic export and apoptosis contribute to regulatory T-cell defects in patients with chronic heart failure. PloS One 6: e24272, 2011.

3. Lichtman AH: The heart of the matter: Protection of the myocardium from T cells. J Autoimmun 45: 90-96, 2013.

4. Haugen E, Gan LM, Isic A, Skommevik T and Fu M: Increased interleukin- 6 but not tumour necrosis factor-alpha predicts mortality in the population of elderly heart failure patients. Exp Clin Cardiol 13: 19-24, 2008.

5. Fritzenwanger M, Jung C, Franz M, Foerster M and Figulla HR: Immunomodulatory effects of cardiotrophin-1 on in vitro cytokine production of monocytes \& CD4 + T-lymphocytes. Indian J Med Res 136: 471-476, 2012.

6. Reifenberg K, Lehr HA, Torzewski M, Steige G, Wiese E, Küpper I, Becker C, Ott S, Nusser P, Yamamura K, et al: Interferon-gamma induces chronic active myocarditis and cardiomyopathy in transgenic mice. Am J Pathol 171: 463-472, 2007.

7. Cheng X, Yu X, Ding YJ, Fu QQ, Xie JJ, Tang TT, Yao R, Chen Y and Liao YH: The Th17/Treg imbalance in patients with acute coronary syndrome. Clin immunol 127: 89-97, 2008.

8. Stumpf C, Lehner C, Yilmaz A, Daniel WG and Garlichs CD: Decrease of serum levels of the anti-inflammatory cytokine interleukin-10 in patients with advanced chronic heart failure. Clin Sci 105: 45-50, 2003.

9. Pudil R, Tichý M, Andrýs C, Rehácek V, Bláha V, Vojácek J and Palicka V: Plasma interleukin-6 level is associated with NT-proBNP level and predicts short and long term mortality in patients with acute heart failure. Acta Medica (Hradec Kralove) 53: 225-228, 2010.

10. Hofmann U and Frantz S: How can we cure a heart 'in flame'? A translational view on inflammation in heart failure. Basic Res Cardiol 108: 356, 2013.

11. Xiang F, Guo X, Chen W, Wang J, Zhou T, Huang F, Cao C and Chen X: Proteomics analysis of human pericardial fluid. Proteomics 13: 2692-2695, 2013.

12. Frohlich GM, Keller P, Schmid F, Wolfrum M, Osranek M, Falk C, Noll G, Enseleit F, Reinthaler M, Meier P, et al: Haemodynamically irrelevant pericardial effusion is associated with increased mortality in patients with chronic heart failure. Eur Heart J 34: 1414-1423, 2013.

13. Hunt SA, Abraham WT, Chin MH, Feldman AM, Francis GS, Ganiats TG, Jessup M, Konstam MA, Mancini DM, Michl K, et al: 2009 focused update incorporated into the ACC/AHA 2005 guidelines for the diagnosis and management of heart failure in adults: A report of the American college of cardiology foundation/American heart association task force on practice guidelines: Developed in collaboration with the International society for heart and lung transplantation. Circulation 119: e391-e479, 2009.

14. Eren M: Diagnosis and treatment of acute and chronic heart failure: What has changed in the new European society of cardiology guideline 2008. Turk Kardiyol Dern Ars 37: 295-300, 2009.

15. Cheitlin MD, Armstrong WF, Aurigemma GP, Beller GA, Bierman FZ, Davis JL, Douglas PS, Faxon DP, Gillam LD, Kimball TR, et al: ACC/AHA/ASE 2003 guideline update for the clinical application of echocardiography: Summary article: A report of the American college of cardiology/American heart association task force on practice guidelines (ACC/AHA/ASE committee to update the 1997 guidelines for the clinical application of echocardiography). Circulation 108: 1146-1162, 2003.

16. Hurst JW: The value of using the entire New York heart association's classification of heart and vascular disease. Clin Cardiol 29: 415-417, 2006.
17. Hunt SA, Abraham WT, Chin MH, Feldman AM, Francis GS, Ganiats TG, Jessup M, Konstam MA, Mancini DM, Michl K, et al: ACC/AHA 2005 guideline update for the diagnosis and management of chronic heart failure in the adult: A report of the American college of cardiology/American heart association task force on practice guidelines (writing committee to update the 2001 guidelines for the evaluation and management of heart failure): Developed in collaboration with the American college of chest physicians and the international society for heart and lung transplantation: Endorsed by the heart rhythm society. Circulation 112: e154-e235, 2005.

18. Li L, Chen W, Zhu Y, Wang X, Jiang DS, Huang F, Wang L, Xiang F, Qin W, Wang Q, et al: Caspase recruitment domain 6 protects against cardiac hypertrophy in response to pressure overload. Hypertension 64: 94-102, 2014.

19. Cao C, Zhu Y, Chen W, Li L, Qi Y, Wang X, Zhao Y, Wan X and Chen $\mathrm{X}$ : IKKe knockout prevents high fat diet induced arterial atherosclerosis and NF-kB signaling in mice. PloS One 8: e64930, 2013.

20. Cao C, Li L, Chen W, Zhu Y, Qi Y, Wang X, Wan X and Chen X: Deficiency of IKKe inhibits inflammation and induces cardiac protection in high-fat diet-induced obesity in mice. Int J Mol Med 34: 244-252, 2014.

21. Ziaeian B and Fonarow GC: Epidemiology and aetiology of heart failure. Nat Rev Cardiol 13: 368-378, 2016.

22. Askevold ET, Nymo S, Ueland T, Gravning J, Wergeland R, Kjekshus J, Yndestad A, Cleland JG, McMurray JJ, Aukrust P and Gullestad L: Soluble glycoprotein 130 predicts fatal outcomes in chronic heart failure: Analysis from the Controlled Rosuvastatin multinational trial in heart failure (CORONA). Circ Heart Fail 6: 91-98, 2013.

23. Kanda $\mathrm{T}$ and Takahashi T: Interleukin-6 and cardiovascular diseases. Jpn Heart J 45: 183-193, 2004.

24. Maeda K, Tsutamoto T, Wada A, Mabuchi N, Hayashi M, Tsutsui T, Ohnishi M, Sawaki M, Fujii M, Matsumoto T and Kinoshita M: High levels of plasma brain natriuretic peptide and interleukin-6 after optimized treatment for heart failure are independent risk factors for morbidity and mortality in patients with congestive heart failure. J Am Coll Cardiol 36: 1587-1593, 2000.

25. Maries L and Manitiu I: Diagnostic and prognostic values of B-type natriuretic peptides (BNP) and N-terminal fragment brain natriuretic peptides (NT-pro-BNP). Cardiovasc J Afr 24: 286-289, 2013

26. Toufan M, Namdar H, Abbasnezhad M, Habibzadeh A, Esmaeili H, Yaraghi S and, Samani Z: Diagnostic values of plasma, fresh and frozen urine NT-proBNP in heart failure patients. J Cardiovasc Thorac Res 6: 111-115, 2014.

27. Chang HR, Hsieh JC, Hsu BG, Wang LY, Chen MY and Wang JH: Inverse association of $\mathrm{N}$-terminal pro-B-type natriuretic peptide with metabolic syndrome in patients with congestive heart failure. PloS One 8: e79096, 2013.

28. Taylor CJ, Roalfe AK, Iles R and Hobbs FD: The potential role of NT-proBNP in screening for and predicting prognosis in heart failure: A survival analysis. BMJ Open 4: e004675, 2014.

29. Szczurek W and Szyguła-Jurkiewicz BS: Oxidative stress and inflammatory markers - the future of heart failure diagmostics? Kardiochir Torakochirurgia Pol 2015: 145-149, 2015.

30. Gruson D, Ahn SA and Rousseau MF: Biomarkers of inflammation and cardiac remodeling: The quest of relevant companions for the risk stratification of heart failure patients is still ongoing. Biochem Med (Zagreb) 21: 254-263, 2011.

31. Hoare GS, Birks EJ, Bowles C, Marczin N and Yacoub MH: In vitro endothelial cell activation and inflammatory responses in end-stage heart failure. J Appl Physiol (1985) 101: 1466-1473, 2006.

32. Ruiz-Ruiz FJ, Ruiz-Laiglesia FJ, Lasierra-Diaz P, Samperiz Legarre P, Morales-Rull JL, Sánchez-Marteles M, Amores M and Perez-Calvo JI: Lack of clinical usefulness of interleukin-6 in long-term follow-up of acutely decompensated heart failure. Singapore Med J 48: 532-536, 2007.

33. Rumalla VK, Calvano SE, Spotnitz AJ, Krause TJ, Hilkert RJ, Lin E and Lowry SF: Alterations in immunocyte tumor necrosis factor receptor and apoptosis in patients with congestive heart failure. Ann Surg 236: 254-260, 2002.

34. Okamoto N, Noma T, Ishihara Y, Miyauchi Y, Takabatake W, Oomizu S, Yamaoka G, Ishizawa M, Namba T, Murakami K, et al: Prognostic value of circulating regulatory $\mathrm{T}$ cells for worsening heart failure in heart failure patients with reduced ejection fraction. Int Heart J 55: 271-277, 2014. 
35. Tang TT, Ding YJ, Liao YH, Yu X, Xiao H, Xie JJ, Yuan J, Zhou ZH, Liao MY, Yao R, et al: Defective circulating CD4CD25+Foxp3+CD127(low) regulatory T-cells in patients with chronic heart failure. Cell Physiol Biochem 25: 451-458, 2010.

36. Gandolfo MT, Jang HR, Bagnasco SM, Ko GJ, Agreda P, Satpute SR, Crow MT, King LS and Rabb H: Foxp $3^{+}$regulatory $\mathrm{T}$ cells participate in repair of ischemic acute kidney injury. Kidney Int 76: 717-729, 2009.

37. Yndestad A: Enhanced expression of inflammatory cytokines and activation markers in T-cells from patients with chronic heart failure. Cardiovasc Res 60: 141-146, 2003.

38. Fritzenwanger M, Jung C, Franz M, Foerster M and Figulla HR: Immunomodulatory effects of cardiotrophin-1 on in vitro cytokine production of monocytes \& CD4 + T-lymphocytes. Indian J Med Res 136: 471-476, 2012.

39. Yndestad A, Damås JK, Oie E, Ueland T, Gullestad L and Aukrust P: Systemic inflammation in heart failure-the whys and wherefores. Heart Fail Rev 11: 83-92, 2006.

40. Cappuzzello C, Di Vito L, Melchionna R, Melillo G, Silvestri L, Cesareo E, Crea F, Liuzzo G, Facchiano A, Capogrossi MC and Napolitano M: Increase of plasma IL-9 and decrease of plasma IL-5, IL-7, and IFN- $\gamma$ in patients with chronic heart failure. J Transl Med 9: 28, 2011.

41. Fukunaga T, Soejima H, Irie A, Sugamura K, Oe Y, Tanaka T, Kojima S, Sakamoto T, Yoshimura M, Nishimura Y and Ogawa H: Expression of interferon-gamma and interleukin-4 production in $\mathrm{CD}^{+} \mathrm{T}$ cells in patients with chronic heart failure. Heart Vessels 22: 178-183, 2007.

42. Fairweather D, Frisancho-Kiss S, Yusung SA, Barrett MA, Davis SE, Gatewood SJ, Njoku DB and Rose NR: Interferon-gamma protects against chronic viral myocarditis by reducing mast cell degranulation, fibrosis and the profibrotic cytokines transforming growth factor-beta 1, interleukin-1 beta, and interleukin-4 in the Heart. Am J Pathol 165: 1883-1894, 2004.
43. Hilfiker-Kleiner D, Shukla P, Klein G, Schaefer A, Stapel B, Hoch M, Müller W, Scherr M, Theilmeier G, Ernst M, et al: Continuous glycoprotein-130-mediated signal transducer and activator of transcription-3 activation promotes inflammation, left ventricular rupture, and adverse outcome in subacute myocardial infarction. Circulation 122: 145-155, 2010.

44. Kinugawa T, Kato M, Yamamoto K, Hisatome I and Nohara R: Proinflammatory cytokine activation is linked to apoptotic mediator, soluble Fas level in patients with chronic heart failure. Int Heart J 53: 182-186, 2012.

45. Júnior ML Jr, Lopes RD, Seelaender MC and Lopes AC: Anti-inflammatory effect of physical training in heart failure role of TNF-alpha and IL-10. Arq Bras Cardiol 93: 643-651, 2009.

46. Amir O, Rogowski O, David M, Lahat N, Wolff R and Lewis BS: Circulating interleukin-10: Association with higher mortality in systolic heart failure patients with elevated tumor necrosis factor-alpha. Isr Med Assoc J 12: 158-162, 2010.

47. Ritschel VN, Seljeflot I, Arnesen H, Halvorsen S, Weiss T, Eritsland $\mathrm{J}$ and Andersen GØ: IL-6 signalling in patients with acute ST-elevation myocardial infarction. Results Immunol 4: $8-13,2013$

48. Pudil R, Tichý M, Andrýs C, Rehácek V, Bláha V, Vojácek J and Palicka V: Plasma interleukin-6 level is associated with NT-proBNP level and predicts short- and long-term mortality in patients with acute heart failure. Acta Medica (Hradec Kralove) 53: 225-228, 2010.

49. Fischer $P$ and Hilfiker-Kleiner D: Role of gp130-mediated signalling pathways in the heart and its impact on potential therapeutic aspects. Br J Pharmacol 153 (Suppl 1): S414-S427, 2008.

50. Richter B, Koller L, Hohensinner PJ,Zorn G, Brekalo M, Berger R, Mörtl D, Maurer G, Pacher R, Huber K, et al: A multi-biomarker risk score improves prediction of long-term mortality in patients with advanced heart failure. Int J Cardiol 168: 1251-1257, 2013. 